Methods This is a prospective observational study of patients with opioid abuse (, who were diagnosed as having GI ulcers or strictures, between January 2016 and December 2018, at Dayanand Medical College and Hospital, Ludhiana, India. The diagnosis was made on the basis of radiology (Computed Tomography Enterography or Magnetic Resonance Enterography) and/or endoscopy (gastroduodenoscopy or ileocolonoscopy). The ulcer/stricture was attributed to opioids once other possible etiologies of GI stricture like non-steroidal antiinflammatory drugs, Crohn's disease, infections, neoplasias, corrosive ingestion, ischaemia and peptic ulcers were excluded. Clinical parameters including presenting complaints, site of disease, haematological and biochemical parameters and treatment given were recorded.

Results During the study period, 18 patients (mean age 38.46 \pm 14.86 years, $100 \%$ males) were diagnosed to have opioidinduced GI ulcers/strictures. Tramadol capsules (77.78\%) followed by poppy husk (22.22\%) were the most common forms of opioid consumption. Iron deficiency anaemia $[n=16$ $(88.89 \%)]$, fatigue $[n=14 \quad(77.78 \%)]$, vomiting $[n=11$ (61.11\%)], pain abdomen and loss of weight $[n=10(55.55 \%)$ each] were the most common presenting complaints. Five $(27.78 \%)$ patients presented with GI bleed. There were two common sites of involvement, gastroduodenal $(n=11,61.11 \%)$ and jejuno-ileal $(n=7,38.88 \%)$. Four $(22.22 \%)$ patients had ulcers whereas $14(77.78 \%)$ patients had strictures on evaluation. Ten $(55.55 \%)$ patients underwent balloon dilatation of stricture, six of whom (60\%) failed to respond and needed surgical intervention. Two patients (11.11\%) were taken up for emergency surgery (without attempting balloon dilatation) as both had deep Forrest Ia duodenal ulcers, refractory to endoscopic management. Results are summarized in table 1 (table 1).

Conclusions Opioid abusers can have gastro-intestinal ulcers and strictures and are difficult to treat with medical/endoscopic therapy.

\section{IDDF2019-ABS-0310 PATTERN OF RESPONSE TO SERIAL FECAL MICROBIOTA TRANSPLANTATION VIA COLONOSCOPIC ROUTE IN PATIENTS WITH ULCERATIVE COLITIS}

Vandana Midha*, Ramit Mahajan, Arshdeep Singh, Varun Mehta, Yogesh Gupta, Kirandeep Kaur, Vikram Narang, Ajit Sood. Dayanand Medical College and Hospital, Ludhiana, India

\subsection{6/gutjnl-2019-IDDFabstracts.228}

Background Fecal microbiota transplantation (FMT) targeting gut microbiome dysbiosis is an emerging therapy for ulcerative colitis (UC) and has been found to be efficacious in multiple randomized trials. However, there is no uniformity in protocols with respect to the route of administration, the frequency of intervention and amount of fecal slurry delivered. We intend to study the pattern of response to serial fecal microbiota transplantation via the colonoscopic route in patients with ulcerative colitis.

Methods A retrospective analysis of patients with active UC (Mayo clinic score $\geq 4$ ), who received multi-session FMT (at weeks $0,2,6,10,14,18$ and 22) via a colonoscopic route, in addition to standard of care, between June 2015 and December 2018 was done. The proportion of patients maintaining steroid-free clinical remission (Mayo score $\leq 2$, all sub scores $\leq 1$ ) and achieving endoscopic remission (endoscopic Mayo score 0) were calculated for each FMT session.

Results One hundred twenty-four patients [mean age 34.84 \pm 11.91 years, $66.93 \%$ males $(n=83)$, mean mayo clinic score $8.13 \pm 2.65]$ who consented for FMT were analysed retrospectively. Proportion of patients maintaining steroid-free clinical remission (Mayo score $\leq 2$, all sub scores $\leq 1$ )as well as achieving endoscopic remission (endoscopic Mayo score 0) increased with serial sessions of FMT $\left(16.6 \%\right.$ after $1^{\text {st }}$ session vs $65.55 \%$ after $7^{\text {th }}$ session for clinical remission and $3.62 \%$ after $1^{\text {st }}$ session vs $37.93 \%$ after $7^{\text {th }}$ session for endoscopic remission). (figure 1)

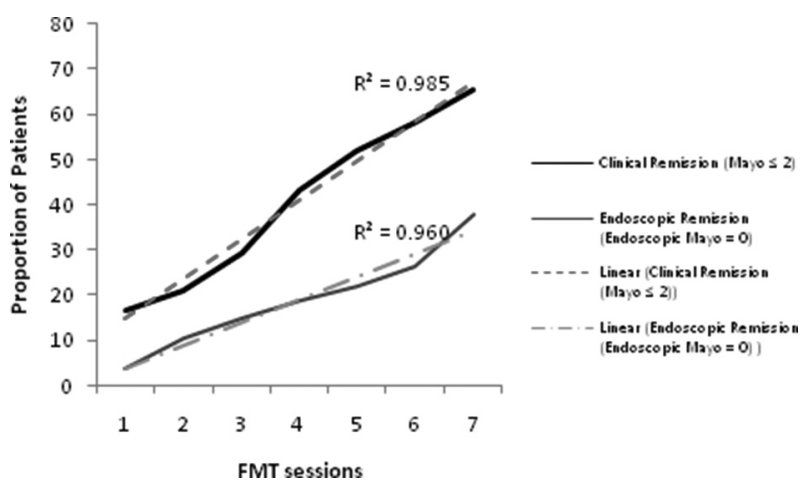

Abstract IDDF2019-ABS-0310 Figure 1

Conclusions With serial colonoscopic FMTs for active ulcerative colitis, the response rates, determined by the maintenance of steroid-free clinical remission and endoscopic remission, gradually increase. Repeated interventions over longer periods may result in a persistent change in gut microbiota composition that is otherwise inherently resilient, resulting in improvement in disease activity indices.

\section{IDDF2019-ABS-0311 EFFICACY AND COST-EFFECTIVENESS OF PREMEDICATION WITH N-ACETYLCYSTEINE DURING UPPER GASTROINTESTINAL ENDOSCOPY EXAMINATION: A SINGLE CENTER, PROSPECTIVE, SINGLE BLINDED, RANDOMIZED CONTROLLED TRIAL}

Lingye Zhang*, Jingling Cheng, Ming Ji, Shanshan Wu, Huihong Zhai, Shutian Zhang. Department of Gastroenterology, Beijing Friendship Hospital, Capital Medical University, China

\subsection{6/gutjnl-2019-IDDFabstracts.229}

Background Detecting gastric cancer in the early stage is the key factor in reducing cancer mortality. Endoscopists can now detect early cancer with the aid of advanced imaging in endoscopy. A major technical obstacle in achieving a good quality of endoscopic examination of the gastric mucosa is the presence of foam and mucus over the mucosal surface. Pronase has become a common anti-mucus agent used for improving visibility during upper gastrointestinal endoscopy examination, especially in eastern Asia. While $\mathrm{N}$-acetylcysteine (NAC) can serve as an alternative mucolytic agent more economically. The aim of this study is to investigate the efficacy and cost-benefit of premedication with NAC during esophagogastroduodenoscopy (EGD). 